\title{
Evolving Materialized Views in Data Warehouse
}

\author{
Chuan Zhang, ${ }^{*}$ Xin Yao, Jian Yang ${ }^{\dagger}$
}

\begin{abstract}
A data warehouse contains multiple views accessed by queries. One of the most important decisions in designing a data warehouse is the selection of materialized views for the purpose of efficiently implementing decision making. The search space for the selection of materialized views is exponentially large, therefore, heuristics have been used to search a small fraction of the space to get a near optimal solution. In this paper, we explore the use of a genetic algorithm for the selection of materialized views based on multiple global processing plans for many queries. Our experimental studies indicate that the genetic algorithm delivers better solutions than some heuristics.
\end{abstract}

\section{Introduction}

A Data Warehouse (DW) is a repository of integrated information available for querying and analysis. DW is an approach to the integration of data from multiple, possibly very large, distributed, heterogeneous databases and other information sources. In this paper, DW data model is based on SPJ (Selection-Projection-Join) model rather than multidimensional model. There are many research issues related to DW [18], among which materialized view selection is one of the DW challenging ones. The related recent work is presented in [17], [8], [2], [7], [10], [13]. The problem is proven to be NP-complete [8]. In this approach, it is to extract and integrate information of interest from each source in advance and store them in a centralized repository. When a query is evaluated, the intermediate result is called view, which is virtual. The view which is stored in advance in repository is called materialized view.

Usually we have the following choices:

- Materializing all the views in the DW can achieve the best performance at the highest cost of maintenance;

- Leaving all the views virtual will have the poorest performance but the lowest maintenance cost;

- If we have some views materialized, and some vir-

${ }^{*}$ Chuan Zhang and Xin Yao are with the School of Computer Science, University College, UNSW, ADFA, Canberra ACT 2600, Australia. E-mail: czhang,xin@cs.adfa.edu.au.

$\dagger$ Jian Yang is with Spatial Information Systems, CSIRO Mathematical and Information Sciences, GPO Box 664, Canberra ACT 2601, Australia. E-mail: Jian.Yang@cmis.csiro.au. tual, especially when there are some shared views on common data involved, then we can achieve an optimal balance between performance and maintenance.

Our problem in this paper can be described as follows: based on multiple queries, select a set of views to be materialized in order that the total query and maintenance cost is minimized. Our problem is different from the multiple query optimization problem. In section 2.4 , we will explain the difference in details.

Most of the work done so far made use of heuristics for selection of materialized views in order to obtain a near optimal solution - the minimum sum of query response time and view maintenance time under some constraint. Especially, in [9], [7], [6], [8], they mainly exploited a greedy algorithm to select materialized views.

The algorithms used in optimization can be classified into four types [16], [4].

1. Deterministic algorithms: Every algorithm in this class constructs a solution step in a deterministic manner, either by applying heuristics or by exhaustive search.

2. Randomized algorithms: Algorithms in this class pursue a completely different approach: First, a set of moves are defined. These moves constitute edges between different solutions of the solution space. Two solutions are connected by an edge if (and only if) they can be transformed into one another by exactly one move. Each of the algorithms performs a random walk along the edges according to certain rules, terminating as soon as no more applicable ones exist or a time limit is exceed. The best solution encountered so far is the result.

3. Genetic algorithm: Genetic algorithms make use of a randomized search strategy very similar to biological evolution in their search for good solutions. Although in this aspect a genetic algorithm resembles randomized algorithms as discussed above, the approach shows enough differences to warrant a consideration of its own. The basic idea is to start with a random population and generate offspring by random crossover and mutation. The "fittest" members of the population(according to the cost function) survive the subsequent selection; the next generation is based 
on these. The algorithm terminates as soon as there is no further improvement or after a predetermined number of generation. The fittest individual found is the solution.

4. Hybrid algorithm: Hybrid algorithms combine the strategies of pure deterministic and pure randomized algorithms: solutions obtained by deterministic algorithms are used as starting points for randomized algorithms or as initial population members for genetic algorithms.

Recently the robustness of genetic algorithm has lead database researchers to consider it to be applied in their own discipline [3], [5], [16].

In this paper, we apply a genetic algorithm to a number of global query processing plans to minimize cost of queries and maintenance.

The rest of the paper is organized as follows: section 2 explains and defines the issues for the selection of materialized views based on multiple processing plans. In section 3 , we present the genetic algorithm for the selection of materialized views based on multiple processing plans. Section 4 presents the experiment results using our evolutionary approach. We conclude in section 5 by analyzing and summarizing our experiments and suggesting some ideas for future work.

\section{Selection of materialized views}

Our problem is related to three optimization issues, i.e, query, multiple queries, the selection of materialized views.

\subsection{Query optimization}

Since join is one of the most expensive operations, for simplicity we only consider join operation. Hence, query optimization is actually join order optimization.

Assume that a database $D$ is given a set of relations $R_{1}, R_{2}, \ldots, R_{m}$. A processing plan for a query $\mathrm{Q}$ is defined as a local processing plan for a query $Q$. A local processing plan is a query graph. Since we only consider join operation, a local processing plan for a query is actually a join binary tree which consists of all relations as its leaves and join operations as its inner nodes. The edges are labeled with the join predicate and the join selectivity. The join predicate maps tuples from the cartesian product of the adjacent nodes to \{false, true , depending whether the tuple is to be included in the result or not. The join selectivity is the ratio included/total number of tuples.

The search space is the set of all local processing plans. Because the structure shape of the processing can be arbitrary, the cardinality of the set is much higher. A point in the search space is one particular plan. Every point of the search space has a cost associated with it. The cost function maps the processing tree to cost.

Since there are lots of methods such as Nested Loop, Sort-Merge, Hash Loop, to perform the join operation, there exist lots of cost functions with respect to the processing tree.

For example, for Nested Loop join with no indices available, each tuple of the outer relation must be checked against every tuple of the inner relation, so we obtain $\operatorname{cost}\left(R_{1} \bowtie R_{2}\right)=\left\|R_{1}\right\| *\left\|R_{2}\right\|$.

Among the solution space, the left-deep processing trees has been of special interest to researchers. The leftdeep tree is a tree where inner relation of each join is a base relation. In this paper, we mainly focus on left-deep tree. For $n$ relations, the number of possible solutions are $m^{*} n$ ! where $m$ is the number of left-deep trees. With the number of relations increasing, $m$ increases rapidly [16].

In Figure 1, the costs of two join trees in (1) may be different due to different join methods. The costs of two join trees in (2) are different as well although the join method and the structure are the same.
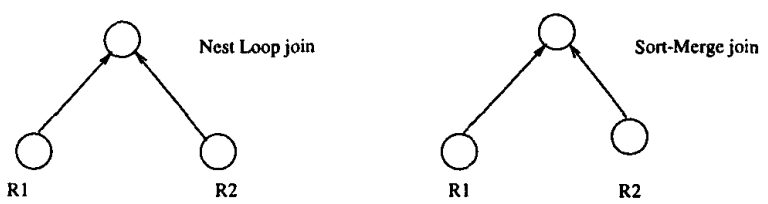

(1)
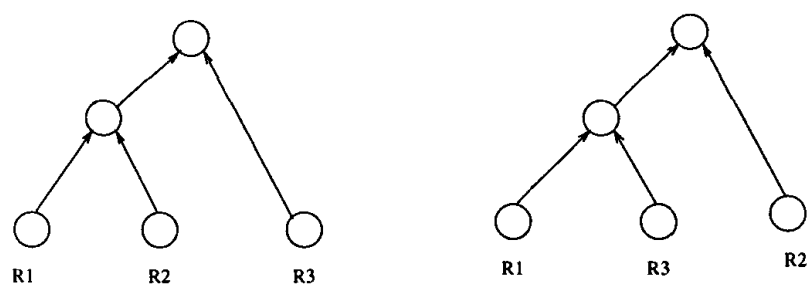

(2)

Figure 1: Join tree illustration

Given a set of processing plans for the query, the goal of query optimization is to find a processing plan within a set of processing plans with lowest cost.

\subsection{Multiple query processing optimization}

A DW is a repository of integrated information available for querying analysis. One issue we have to deal with is multiple query processing. In [11], [14], a systematic look at the problem has been presented.

Assume that a set of queries $\mathrm{Q}=Q_{1}, Q_{2}, \ldots, Q_{n}$ is given. For every query $Q_{i}$, there exist at least one pro- 
cessing plan. We called it a local processing plan. A global/multiple processing plan for $\mathrm{Q}$ corresponds to a plan that provides a way to compute the results of all $n$ queries. A global/multiple access plan can be constructed by choosing one plan for each query and then merging them together. The locally optimal plans is referred to as the cost plans for processing each query $Q_{i}$ individually. This corresponds to the join order optimization. The globally optimal plan is referred to as the global processing plans by merging the common parts of individual local plans.

The Multiple Query optimization(MQO) problem is as follows: Given $n$ sets of processing plans $P_{1}, P_{2}, \ldots, P_{n}$, with $P_{i}=\left\{P_{i 1}, P_{i 2}, \ldots, P_{i k_{i}}\right\}$ being the set of possible plans for query $Q_{i}, 1 \leq i \leq n, k_{i}$ is the number of local processing plans for $Q_{i}$. Find a global $/$ multiple processing plan by selecting one plan from each $P_{i}$ such that the cost (query cost) of global/multiple processing plan is minimized.

In general, the union of the locally optimal plan is different from the globally optimal plan. Hence, we cannot find the globally optimal plan by simply combining the locally optimal plan.

In our problem, the multiple query optimization is somewhat different from that presented in [11], [14], we look into all possible combinations of individual query plans by sharing the common parts and then based on these combinations select the materialized views.

When combining multiple query processing plans, i.e. multiple join trees, the produced global processing plan should be a DAG (Directed Acyclic Graph) not a tree. This is shown in Figure 2.

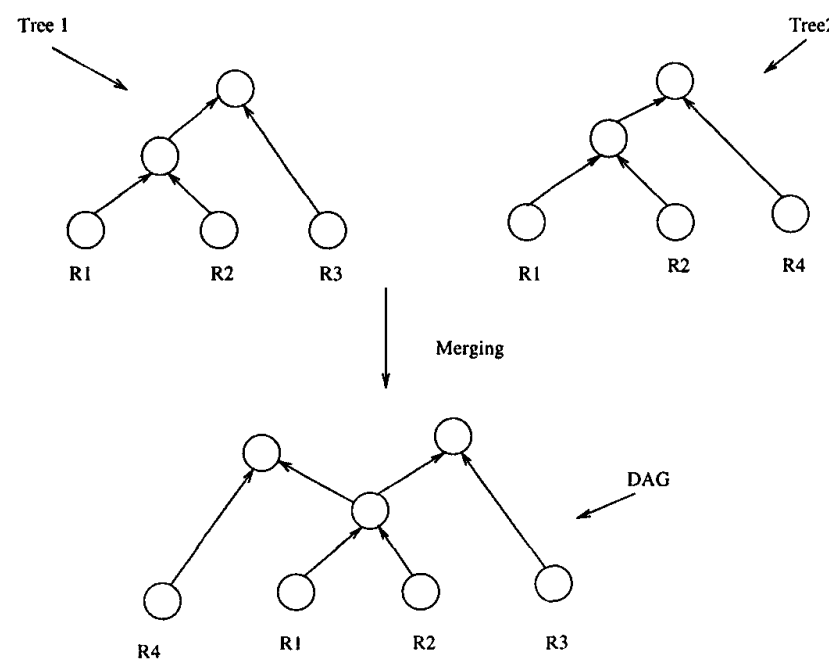

Figure 2: A global processing plan should be a DAG

\subsection{The materialized view selection optimization}

In the DW approach, selected information is extracted in advance and stored in a repository. A DW can therefore be seen as a set of materialized views defined over the sources.

Now the problem we are dealing with is how to select the views to be materialized so that the cost of query processing and view maintenance for all the nodes in a global processing plan is minimized.

An obvious approach is to apply the exhaustive search algorithm for materialized view selection on the set of queries. However this approach is impractical if the search space is big. Many researchers take the heuristic way to trim the search space in order to get the results quickly, but by this way, only a near-optimal solution can be achieved. Compared to heuristic optimization, genetic algorithms have many advantages such as searching from a population of points using probabilistic transition rules. In order to avoid exhaustive search in the whole solution space and obtain a better solution than that obtained by heuristic methods, we will apply a genetic algorithm to our problem.

In the next section, we will present a brief view of cost model used for selection of materialized views, then we will explain what the genetic algorithm is and how to apply it to our problem.

\subsection{The cost model of materialized view selection}

As described in last section, a global processing plan is a DAG.

Let $f_{q}, f_{u}$ be query frequency and maintenance frequency respectively. $q_{i}$ denotes the $i$ th query.

We use the linear cost model [9] to calculate the cost of query $Q$ : The cost of answering $Q$ is the number of rows present in the table for that query $Q_{A}$ used to construct $Q$.

Let $M$ be a set of materialized views. The goal is to find the set $M$, such that the total cost of query processing and maintenance of $\mathrm{M}$ is minimized.

Let $C_{q_{i}}(M)$ be the cost to compute $q_{i}$ from the set of materialized views M. $C_{m}(v)$ be the cost of maintenance when $v$ is materialized. Then the total query processing cost is $\sum_{q_{i} \in Q} f_{q_{i}} C_{q_{i}}(M)$. The total maintenance cost is $\sum_{v \in M} f_{u} C_{m}(v)$. The total cost of one solution, i.e., one set of materialized views $\mathrm{M}$, is

$\sum_{q_{i} \in Q} f_{q_{i}} C_{q_{i}}(M)+\sum_{v \in M} f_{u} C_{m}(v)$

Given a global processing plan, the objective is to minimize the above cost.

Given $n$ sets of local processing plans $P_{1}, P_{2}, \ldots, P_{n}$, with $P_{i}=\left\{P_{i 1}, P_{i 2}, \ldots, P_{i k_{i}}\right\}$ being the set of possible plans for query $Q_{i}, 1 \leq i \leq n, k_{i}$ is the number of local processing plans for $Q_{i}$. Find a global processing plan by "merging" $n$ local processing plans (one out of each set $P_{i}$ ). Within this Global Processing plan, select a 
set of views to be materialized such that total_cost is minimized.

What distinguishes our problem from MQO (Multiple Query Optimization) is the following [19]:

1. MQO is to find an optimal processing plan for multiple queries executed at the same time by sharing some temporary results which are common subexpressions, while our problem is to find a set of relations(which can be any intermediate result from query processing), to be materialized so that the total cost(query processing plus view maintenance) is minimized.

2. In MQO, a global processing plan is derived from the idea that temporary result sharing should be less expensive compared to a serial execution of queries. However, this may not be true for every possible database state. For example, sharing temporary result may prove to be a bad decision when indices on base relations are defined. The cost of processing a selection through an index or through an existing temporary result clearly depends on the size of these two structures. While in our problem, if an intermediate result is materialized, we can establish a proper index on it afterwards if necessary. Therefore, it is guaranteed that there is a performance gain if an intermediate result is materialized. If the intermediate result happens to be a common subexpression which can be shared by more than one query, then there is a view maintenance gain as well.

3. In MQO, the ultimate goal is to achieve the best performance, while our problem has to take into consideration of both query and view maintenance cost.

4. In MQO, the input is a set of queries and the output is a global optimal plan. In our problem, however, the input are a set of global queries and their access frequencies, and a set of base relations and their update frequencies. The output is one or more global processing plans with minimized total query and maintenance cost.

Just as we cannot guarantee to get the globally optimal processing plan by the union of the locally optimal plan, we cannot guarantee to get the optimal solution of the selection of materialized views based on the optimal global/multiple processing plan.

For example, an optimal global processing plan may have optimal query cost $333 m$. $m$ denotes million. Based on this global processing plan, we may obtain the total cost (query cost plus maintenance cost) is $310 \mathrm{~m}$. But with a non-optimal global processing plan that has query cost $350 \mathrm{~m}$, we might get the total cost (query cost plus maintenance cost) $300 \mathrm{~m}$, which is less than $310 \mathrm{~m}$.

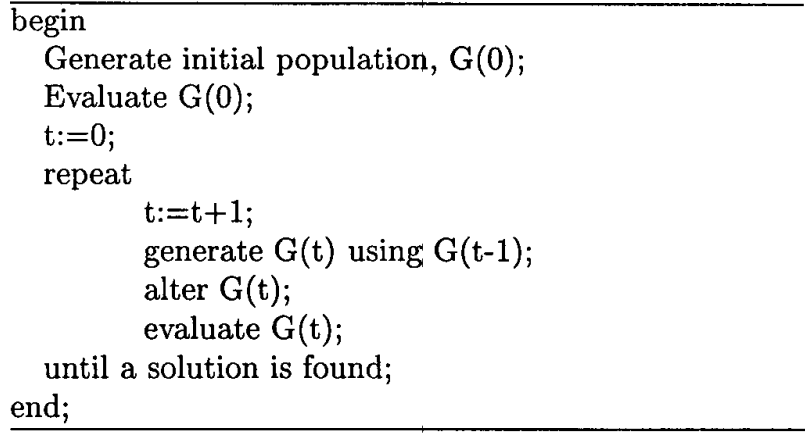

Figure 3: An abstract view of Genetic Algorithm

This illustrates that optimization of selection of materialized views is difficult.

To find the optimal solution for selection of materialized views, one could enumerate all possible combinations of locally processing plans for $m$ queries, but there will be $n_{1} !^{*} n_{2} !^{*} \ldots n_{m}$ ! combinations. Based on every produced global processing plan, supposed that there are $n$ nodes, finding the optimal solution for selection of materialized views will be $\mathrm{O}\left(2^{n}\right)[19]$.

\section{Evolving materialized views}

\subsection{Genetic algorithm}

One of differences between genetic algorithms and other heuristic techniques is that genetic algorithms operate on a population of individuals, not a single individual. Every population is called a generation. A single solution is called a phenotype represented by a single individual. Solutions are represented as chromosomes, which is composed of genes that can take different values (alleles). A genetic algorithm creates an initial generation, $\mathrm{G}(0)$, and for each generation, $G(t)$, generates a new one, $G(t+1)$. An abstract view of the algorithm is shown in Figure 3.

Each problem should have its own solutions represented as chromosomes by an appropriate encoding. Selection, crossover, and mutation, are applied to successive populations to create new populations. In other words these three operators are applied to $G(t-1)$ to generate $G(t)$ as shown in Figure 3. In order to get the best solution, a lot of generations may be evolved. Several stopping criteria exist for the algorithm. For example, the algorithm may be halted when all solutions in a generation are identical. An alternative would halt after a fixed number of evaluations and take the best solution found.

\subsection{The representation of our solution}

Representation is a key issue in genetic algorithms because genetic algorithms manipulate a coded representation of the problem. 
Given $n$ queries $Q_{1}, Q_{2}, \ldots Q_{n}$, the fixed length of each individual is $n$, where $n$ is the number of queries. An individual looks like: $P_{1 i}, P_{2 j}, \ldots, P_{n k}$, where $P_{m n}$ indicates the $n$th processing plan for query $Q_{m}$, represented by an integer number.

With the set of processing plans for one query, we order the processing plans according to certain criteria like ascending order of cost, and then obtain an array of integer numbers corresponding to the processing plans.

For example, there are three queries: $Q_{1}, Q_{2}, Q_{3}$ and the respective number of local processing plans are 12 , 120,120 . The string of $\{[4],[89],[70]\}$ represents the 4 th processing plan for $Q_{1}$, the 89th processing plan for $Q_{2}$, and the 70th processing plan for $Q_{3}$. [4] is from [1..12], [89] is from [1..120], and [70] is from [1..120].

\subsection{Mapping cost function in our problem to fit- ness function in genetic algorithm}

Since the objective in our cost model is stated as the minimization of sum of query cost and maintenance cost and the fitness function of a genetic algorithm is naturally stated as maximization, there should be a transformation from our cost function to the fitness function. We use a common used transformation in genetic algorithms:

$$
f(x)= \begin{cases}C_{\max }-\mathrm{c}(\mathrm{x}) & \text { when } c(x)<C_{\max } \\ 0 & \text { otherwise }\end{cases}
$$

where $c(x)$ denotes the cost function and $f(x)$ is the fitness function. There are a lot of ways to choose the coefficient $C_{\max }$. $C_{\max }$ may be taken as an input coefficient, as the largest $c(x)$ value observed so far, as the largest $c(x)$ value in the current population, or the largest of the last $k$ generations.

With this transformation, we can reach our goal. The less the cost function, the more the fitness function.

An individual is evaluated as follows:

1. Integrate/merge local processing plans by merging common parts among the local processing plans.

2. Apply a genetic algorithm or other heuristics to obtain the set of materialized views in order to minimize the total cost (query cost plus maintenance cost) of this global processing plan.

Note that: the genetic algorithm here is applied to a fixed global processing plan to select materialized views. It is different from the genetic algorithm used to optimize global processing plans. We will refer to it as a lower level genetic algorithm compared with the genetic algorithm applied to multiple queries. The heuristic here is one used in [19]. It was applied to a fixed global processing plan to get the set of materialized views.

3. Transform this optimal cost to the fitness of the individual.

\subsection{Crossover, Mutation and Selection}

\section{Crossover}

The crossover operator attempts to swap partially good solutions in order to get better results. One point crossover is used in our genetic algorithm.

For example, given two individuals:

$L_{1}=[4][20][30][[10][99]$, and

$L_{2}=[5][30][21] \mid[40][80]$

where the symbol | represents the position of crossover applied. The results of crossover are:

$L_{1}^{\prime}=[4][20][30][40][80]$, and

$L_{2}^{\prime}=[5][30][21][10][99]$.

\section{Mutation}

The mutation operator is a means of the occasional random alteration of the value of a gene. It introduces new features that may not present in any member of the population. The mutation is performed on a gene by gene basis. For example, assume that the 3rd gene in the individual $L_{1}=[4][20][30] \mid[10][99]$ is selected for mutation. Suppose that the 3rd query has 120 processing plans, a random number between 1 and 120 would be produced. Suppose the random number is 16 . Then the individual $L_{1}$ after this mutation would be

$L_{1}^{\prime}=[4][20][16] \mid[10][99]$

\section{Selection}

The selection is a process in which individuals are reproduced according to their fitness. Individual with higher fitness values have higher chance to survive. Tournament selection is used in our experiment. Tournament selection means holding tournaments between a number of individuals and selecting the best member of a tournament group for survival to the next generation. The tournament members are typically chosen uniformly at random, and the tournament size (number of individuals involved per tournament) are usually small, ranging from two to ten individuals. If the size is equal to 1 , that means no selection at all. For many applications in genetic programming values (6..10) have been recommended [1]. To our limited experience, we took tournament size, ranging from 4 to 7 .

\section{Experimental studies}

All experiments were performed under SUNOS 5.5. The simulation software was built on the basis of the Simple Genetic Algorithm [15] and GAlib [12]. Based on the 
Simple Genetic Algorithm program [15] which is a Clanguage translation and extension of the original Pascal SGA code presented in [4], we developed the lower level genetic algorithm. Since GAlib [12] is a library about genetic algorithms, it includes almost everything about representation and genetic operators, we developed the higher level genetic algorithm based on GAlib.

For simplicity, query processing plans used in our experiments are generated as a set of left-deep binary trees. It has been argued that good solutions are likely to exist among these trees [16]. The experiments were run over randomly generated queries. These queries share at least two relations. For our experiments, we have generated up to 60 queries. Each query has from 6 to 720 different query processing plans. The solution space for such problems is huge. For example, with 10 queries, there are $720^{10}$ global processing plans. With each global processing plan, there are 40 join nodes in average, the complexity of selecting materialized views is $\mathrm{O}\left(2^{40}\right)$. Hence, the total solution space is $720^{10 *}\left(\mathrm{O}\left(2^{40}\right)\right)$. Using this design, experimental results reliably show the performance of the different approach.

In order to study the behaviour of our algorithm, we compare it with the following intuitive optimization heuristic method:

1. We create global processing plans by merging all the local optimal plans. Note that, there may be more than one local optimal plans for one query. Global processing plans are the single combination of different local optimal plans.

2. Based on these global processing plans, we apply the genetic algorithm to select materialized views.

3 . Compare the total cost (query cost plus maintenance cost) of each global processing plan and select the one which gives the lowest cost.

In Figure 4, GA1 denotes the higher level genetic algorithm applied to multiple queries, GA2 denotes the lower level genetic algorithm applied to a single global processing plan, $\mathrm{H} 1$ denotes the intuitive heuristic described above, H2 denotes that one used in [19]. H1 is different from $\mathrm{H} 2$. $\mathrm{H} 1$ is used for the selection of processing plans based on multiple queries. $\mathrm{H} 2$ is used for the selection of materialized views on a fixed global processing plan.

We have experimented with different combinations of algorithms to figure out the best one. We have the following combinations: GA1-GA2 means that a higher level genetic algorithm was applied to multiple queries and a lower level genetic algorithm was applied to every fixed global processing plan; GA1-H2-GA2 denotes that a genetic algorithm was applied to multiple queries and to every fixed global processing plan the heuristic $\mathrm{H} 2$ was applied, finally, to the best individual, apply genetic algorithm GA2 to get the final results. GA1-H2 means that genetic algorithm was applied to multiple queries and to every fixed global processing plan the heuristic $\mathrm{H} 2$ was applied. $\mathrm{H} 1-\mathrm{H} 2$ means that $\mathrm{H} 1$ was applied to multiple queries and to every fixed global processing plan the heuristic $\mathrm{H} 2$ was applied. H1-GA2 denotes that $\mathrm{H} 1$ was applied to multiple queries and to every fixed global processing plan genetic algorithm was applied.

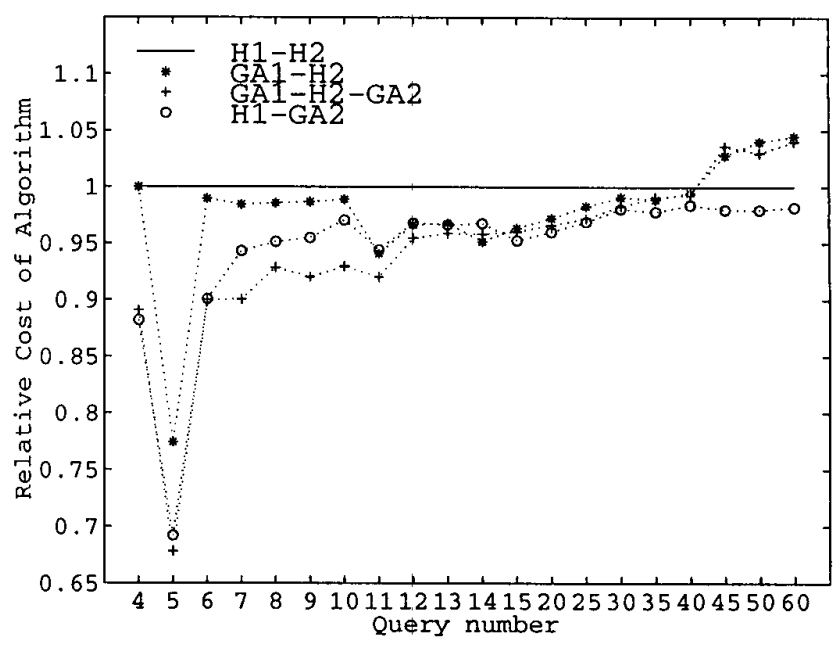

Figure 4: Graph of compared results

Figure 4 shows the comparison among different combinations algorithms. The costs are normalized using $\mathrm{H} 1-\mathrm{H} 2$ algorithm as the reference.

Since GA1-GA2 costs days of time, it is not included in Figure 4.

From the results shown in Figure 4, we can conclude that with small number of queries, GA1-H2-GA2 appears to be the best result among those combinations of algorithms. H1-H2 got the fastest evaluation time. With the increase of queries, H1-GA2 still keep obtaining the best results with time cost increasing slightly. Since the total cost increases hugely as query number increases, a little difference in the comparison of the results will result in a large amount of total cost saving. With large number of queries, H1-GA2 appears to be a good choice.

\section{Conclusion}

The selection of materialized views based on multiple query processing plans is a combinatorial optimization problem. In this study, a genetic algorithm was developed to optimize the selection of materialized views. The performance of the proposed genetic algorithm was compared with other heuristics. The performance of the combination of a genetic algorithm and other heuristics is also studied. The results show that combination of genetic algorithm and heuristic is a good approach. Considering the performance and the evaluation cost, the 
combination of genetic algorithm and heuristic is better than using only genetic algorithms or only heuristics. There is always a trade-off between the total cost of query plus maintenance and the optimization cost. If the DW is real-time, and queries change frequently, genetic algorithms may be improper to this kind of application. If the DW is relatively stable, and the queries do not change frequently, then it is worthwhile to apply the genetic algorithm.

From the results shown in figure 4 , we can conclude that genetic algorithm works better than heuristics. In the future, we should explore other combinatorial optimization algorithm such as simulated annealing, and compare them with genetic algorithm.

\section{Bibliography}

[1] Thomas Back, David B Fogel, and Zbigniew Michalewicz. Handbook of evolutionary computation. IOP Publishing and Oxford University Press, 1997.

[2] Elen Baralis, Stefano Paraboschi, and Ernest Teniente. Materialized view selection in a multidimensional database. Proceedings of the 23rd VLDB Conference, Athens, Greece, pages 156-165, 1997.

[3] Kristin Bennett, Michael C. Ferris, and Yannis Ioannidis. A genetic algorithm for database query optimization. Technical Report TR1004, University of Wisconsin, Madison(WI), 1991.

[4] D.E. Goldberg. Genetic algorithms in search, optimization and machine learning. Addison Wesley, Reading(MA), 1989.

[5] Michael Gregory. Genetic algorithm optimisation of distributed database queries. Proc. of ICEC'98, pages $271-276,1998$.

[6] Himanshu Gupta. Selection of views to materialize in a data warehouse. Proceedings of the International Conference on Data Engineering, Burmingham, U.K., pages 98-112, April, 1997.

[7] Himanshu Gupta, Venky Harinarayan, and Anand Rajaraman. Index selection for olap. Proceedings of the International Conference on Data Engineering, pages 208-219, 1997.

[8] Himanshu Gupta and Inderpal Singh Mumick. Selection of views to materialize under a maintenance cost constraint. Proceedings of the International Conference on Data Engineering, 1998.

[9] Venky Harinarayan, Anand Rajaraman, and Jeffrey D. Ullman. Implementing data cubes efficiently. ACM SIGMOD International Conference on Management of Data, pages 205-227, 1996.

[10] Wilburt Juan Labio, Dallan Quass, and Brad Adelberg. Physical database design for data warehouses. Proceedings of the International Conference on Data Engineering, pages 277-288, 1997.
[11] Timos K.Sellis. Multiple-query optimization. $A C M$ Transactions on Database Systems, 13(1):23-52, March 1988.

[12] Massachusetts Institute of Technology. Galib: A c++ genetic algorithms library. http://lancet.mit.edu/galib-2.4/GAlib.html, v2.4.

[13] K.A. Ross, Divesh Srivastava, and S. Sudarshan. Materialized view maintenance and integrity constraint checking: Trading space for time. Proceedings of the ACM SIGMOD, pages 447-458, 1996.

[14] Sellis Timos Shim Kyuseok and Dana Nau. Improvements on a heuristic algorithm for multiplequery optimization. Data and Knowledge Engineering, 12:197-222, 1994.

[15] Robert E Smith, David E. Goldberg, and Jeff A. Earickson. SGA-C: A C-language implementation of simple genetic algorithm. TCGA Report No. 91002, March,1994.

[16] Michael Steinbrunn, Guido Moerkotte, and Alfons Kemper. Heuristic and randomized optimization for the join ordering problem. $V L D B, 6(3): 191-208$, 1997.

[17] Dimitri Theodoratos and Timos Sellis. Data warehouse configuration. Proceedings of the 23rd VLDB Conference Athens, Greece, 1997, pages 126-135, 1997.

[18] Jennifer Widom. Research problems in data warehouse. Proceedings of 4 th International Conference on Information and Knowledge Management, pages 25-30, 1995.

[19] Jian Yang, Kamalakar Karlapalem, and Qing Li. Algorithm for materialized view design in data warehousing environment. $V L D B^{\prime} 97$, pages $20-40$, 1997. 